\title{
Normality of numbers generated by the values of polynomials at primes
}

\author{
by \\ Yoshinobu NAkAi (Kofu) and IEkATA Shiokawa (Yokohama) \\ To the memory of Norikata Nakagoshi
}

1. Introduction. Let $r \geq 2$ be a fixed integer and let $\theta=0 . a_{1} a_{2} \ldots$ be the $r$-adic expansion of a real number $\theta$ with $0<\theta<1$. Then $\theta$ is said to be normal to base $r$ if, for any block $b_{1} \ldots b_{l} \in\{0,1, \ldots, r-1\}^{l}$,

$$
n^{-1} N\left(\theta ; b_{1} \ldots b_{l} ; n\right)=r^{-l}+o(1)
$$

as $n \rightarrow \infty$, where $N\left(\theta, b_{1} \ldots b_{l} ; n\right)$ is the number of indices $i \leq n-l+1$ such that $a_{i}=b_{1}, a_{i+1}=b_{2}, \ldots, a_{i+l-1}=b_{l}$. Let $(m)_{r}$ denote the $r$ adic expansion of an integer $m \geq 1$. For any infinite sequence $\left\{m_{1}, m_{2}, \ldots\right\}$ of positive integers, we consider the number $0 .\left(m_{1}\right)_{r}\left(m_{2}\right)_{r} \ldots$ whose $r$-adic expansion is obtained by the concatenation of the strings $\left(m_{1}\right)_{r},\left(m_{2}\right)_{r}, \ldots$ of $r$-adic digits, which will be written simply as $0 . m_{1} m_{2} \ldots(r)$.

Copeland and Erdős [1] proved that the number $0 . m_{1} m_{2} \ldots(r)$ is normal to base $r$ for any increasing sequence $\left\{m_{1}, m_{2}, \ldots\right\}$ of positive integers such that, for every positive $\varrho<1$, the number of $m_{i}$ 's up to $x$ exceeds $x^{\varrho}$ provided $x$ is sufficiently large. In particular, the normality of the number

$$
0.23571113 \ldots(r)
$$

defined by the primes was established. Davenport and Erdős [2] proved that the number

$$
0 . f(1) f(2) \ldots f(n) \ldots(r)
$$

is normal to base $r$, where $f(x)$ is any nonconstant polynomial taking positive integral values at all positive integers.

In this paper, we prove the following

Theorem. Let $f(x)$ be as above. Then the number

$$
\alpha(f)=0 . f(2) f(3) f(5) f(7) f(11) f(13) \ldots(r)
$$

1991 Mathematics Subject Classification: 11K, 11L. 
defined by the values of $f(x)$ at primes is normal to base $r$. More precisely, for any block $b_{1} \ldots b_{l} \in\{0,1, \ldots, r-1\}^{l}$, we have

$$
n^{-1} N\left(\alpha(f) ; b_{1} \ldots b_{l} ; n\right)=r^{-l}+O\left(\frac{1}{\log n}\right)
$$

as $n \rightarrow \infty$, where the implied constant depends possibly on $r, f$, and $l$.

2. Preliminary of the proof of the Theorem. Let $\alpha(f)=$ $0 . a_{1} a_{2} \ldots a_{n} \ldots$ be the $r$-adic expansion of the number $\alpha(f)$ given in the Theorem. Then each $a_{n}$ belongs to the corresponding string $\left(f\left(p_{\nu}\right)\right)_{r}$, where $p_{\nu}$ is the $\nu$ th prime and $\nu=\nu(n)$ is defined by

$$
\sum_{i=1}^{\nu-1}\left(\left[\log _{r} f\left(p_{i}\right)\right]+1\right)<n \leq \sum_{i=1}^{\nu}\left(\left[\log _{r} f\left(p_{i}\right)\right]+1\right) .
$$

Here $[t]$ denotes the greatest integer not exceeding the real number $t$. We put $x=x(n)=p_{\nu(n)}$, so that

$$
\begin{aligned}
n & =\sum_{p \leq x} \log _{r} f(p)+O(\pi(x))+O\left(\log _{r} f(x)\right) \\
& =\frac{d x}{\log r}+O\left(\frac{x}{\log x}\right),
\end{aligned}
$$

where $d \geq 1$ is the degree of the polynomial $f(t), p$ runs through prime numbers, and $\pi(x)$ is the number of primes not exceeding $x$. We used here the prime number theorem:

$$
\pi(x)=\operatorname{Li} x+O\left(\frac{x}{(\log x)^{G}}\right),
$$

where $G$ is a positive constant given arbitrarily and

$$
\operatorname{Li} x=\int_{2}^{x} \frac{d t}{\log t} .
$$

Then we have

$$
\begin{aligned}
N\left(\alpha(f) ; b_{1} \ldots b_{l} ; n\right) & =\sum_{p \leq x} N\left(f(p) ; b_{1} \ldots b_{l}\right)+O(\pi(x))+O\left(\log _{r} f(x)\right) \\
& =\sum_{p \leq x} N\left(f(p) ; b_{1} \ldots b_{l}\right)+O\left(\frac{n}{\log n}\right)
\end{aligned}
$$

with $x=x(n)=p_{\nu(n)}$.

Let $j_{0}$ be a large constant. Then for each integer $j \geq j_{0}$, there is an integer $n_{j}$ such that

$$
r^{j-2} \leq f\left(n_{j}\right)<r^{j-1} \leq f\left(n_{j}+1\right)<r^{j} .
$$


We note that

$$
n_{j} \gg \ll r^{j / d}
$$

and that $n_{j}<n \leq n_{j+1}$ if and only if the $r$-adic expansion of $f(n)$ is of length $j$; namely,

$$
(f(n))_{r}=c_{j-1} \ldots c_{1} c_{0} \in\{0,1, \ldots, r-1\}^{j}, \quad c_{j-1} \neq 0 .
$$

For any $x>r^{j_{0}}$, we define an integer $J=J(x)$ by

$$
n_{J}<x \leq n_{J+1}
$$

so that

$$
J=\log _{r} f(x)+O(1) \gg \ll \log x .
$$

Let $n$ be an integer with $n_{j}<n \leq n_{j+1}$ and $j_{0}<j \leq J$, so that $(f(n))_{r}$ can be written as in (3). We denote by $N^{*}\left(f(n) ; b_{1} \ldots b_{l}\right)$ the number of occurrences of the block $b_{1} \ldots b_{l}$ appearing in the string $\underbrace{0 \ldots 0}_{J-j} c_{j-1} \ldots c_{1} c_{0}$
of length $J$. Then we have

$$
\begin{aligned}
0 & \leq \sum_{p \leq x} N^{*}\left(f(p) ; b_{1} \ldots b_{l}\right)-\sum_{p \leq x} N\left(f(p) ; b_{1} \ldots b_{l}\right) \\
& \leq \sum_{j=j_{0}+1}^{J-1}(J-j)\left(\pi\left(n_{j+1}\right)-\pi\left(n_{j}\right)\right)+O(1) \\
& \leq \sum_{j=j_{0}+1}^{J-1} \pi\left(n_{j+1}\right)+O(1) \ll \sum_{j=1}^{J-1} \frac{r^{j / d}}{J} \ll \frac{x}{\log x}
\end{aligned}
$$

and so

$$
N\left(\alpha(f) ; b_{1} \ldots b_{l} ; n\right)=\sum_{p \leq x} N^{*}\left(f(p) ; b_{1} \ldots b_{l}\right)+O\left(\frac{n}{\log n}\right)
$$

with $x=x(n)=p_{\nu(n)}$.

We shall prove in Sections 4 and 5 that

$$
\sum_{p \leq x} N^{*}\left(f(p) ; b_{1} \ldots b_{l}\right)=r^{-l} \pi(x) \log _{r} f(x)+O\left(\frac{x}{\log x}\right)
$$

which, combined with (5) and (2), yields (1). 


\section{Lemmas}

Lemma $1([9 ; 4.19])$. Let $F(x)$ be a real function, $k$ times differentiable, and satisfying $\left|F^{(k)}(x)\right| \geq \lambda>0$ throughout the interval $[a, b]$. Then

$$
\left|\int_{a}^{b} e(F(x)) d x\right| \leq c(k) \lambda^{-1 / k} .
$$

Lemma 2 ([3; p. 66, Theorem 10]). Let

$$
F(t)=\frac{h}{q} t^{d}+\alpha_{1} t^{d-1}+\ldots+\alpha_{k},
$$

where $h, q$ are coprime integers and $\alpha_{i}$ 's are real. Suppose that

$$
(\log x)^{\sigma} \leq q \leq x^{d}(\log x)^{-\sigma},
$$

where $\sigma>2^{6 d}\left(\sigma_{0}+1\right)$ with $\sigma_{0}>0$. Then

$$
\left|\sum_{p \leq x} e(F(p))\right| \leq c(d) x(\log x)^{-\sigma_{0}}
$$

as $x \rightarrow \infty$, where $p$ runs through the primes.

Lemma 3 ([3; p. 2, Lemma 1.3 and p. 5, Lemma 1.6]). Let

$$
F(x)=b_{0} x^{d}+b_{1} x^{d-1}+\ldots+b_{d-1} x+b_{d}
$$

be a polynomial with integral coefficients and let $q$ be a positive integer. Let $D$ be the greatest common divisor of $q, b_{0}, b_{1}, \ldots$, and $b_{d-1}$. Then

$$
\left|\sum_{n=1}^{q} e\left(\frac{F(n)}{q}\right)\right| \leq d^{3 \omega(q / D)} D^{1 / d} q^{1-1 / d}
$$

as $q \rightarrow \infty$, where $\omega(n)$ is the number of distinct prime divisors of $n$.

Lemma 4 ([6; Corollary of Lemma]). Let $F(x)$ be a polynomial with real coefficients with leading term $A x^{d}$, where $A \neq 0$ and $d \geq 2$. Let $a / q$ be $a$ rational number with $(a, q)=1$ such that $|A-a / q|<q^{-2}$. Assume that

$$
(\log Q)^{H} \leq q \leq Q^{d} /(\log Q)^{H},
$$

where $H>d^{2}+2^{d} G$ with $G \geq 0$. Then

$$
\left|\sum_{1 \leq n \leq Q} e(F(n))\right| \ll Q(\log Q)^{-G} .
$$

Lemma 5 ([7; Theorem], cf. [8; Theorem 1]). Let $f(t)$ and $b_{1} \ldots b_{l}$ be as in Theorem. Then

$$
\sum_{n \leq y} N\left(f(n) ; b_{1} \ldots b_{l}\right)=r^{-l} y \log _{r} f(y)+O(y)
$$

as $y \rightarrow \infty$, where the implied constant depends possibly on $r, f$, and $l$. 
4. Proof of the Theorem. We have to prove the inequality (6). We write

$$
\sum_{p \leq x} N^{*}\left(f(p) ; b_{1} \ldots b_{l}\right)=\sum_{p \leq x} \sum_{m=l}^{J} I\left(\frac{f(p)}{r^{m}}\right)
$$

where

$$
I(t)= \begin{cases}1 & \text { if } \sum_{k=1}^{l} b_{k} r^{-k} \leq t-[t]<\sum_{k=1}^{l} b_{k} r^{-k}+r^{-l}, \\ 0 & \text { otherwise. }\end{cases}
$$

There are functions $I_{-}(t)$ and $I_{+}(t)$ such that $I_{-}(t) \leq I(t) \leq I_{+}(t)$, having Fourier expansion of the form

$$
I_{ \pm}(t)=r^{-l} \pm J^{-1}+\sum_{\substack{\nu=-\infty \\ \nu \neq 0}}^{\infty} A_{ \pm}(\nu) e(\nu t)
$$

with

$$
\left|A_{ \pm}(\nu)\right| \ll \min \left(|\nu|^{-1}, J \nu^{-2}\right)
$$

where $e(x)=e^{2 \pi i x}([10$; Chap. 2, Lemma 2]). We choose a large constant $c_{0}$ and put

$$
M=\left[c_{0} \log _{r} J\right] .
$$

Then it follows that

(8) $\sum_{p \leq x} N^{*}\left(f(p) ; b_{1} \ldots b_{l}\right)$

$$
\begin{aligned}
& \lesseqgtr\left(\sum_{l \leq m \leq d M}+\sum_{d M<m \leq J-M}+\sum_{J-M<m \leq J}\right) \sum_{p \leq x} I_{ \pm}\left(\frac{f(p)}{r^{m}}\right) \\
& =\sum_{1}+\frac{\pi(x)}{r^{l}}(J-d M)+\sum_{2}+\sum_{3}+O(\pi(x)),
\end{aligned}
$$

where $d$ is the degree of the polynomial $f(x)$,

$$
\begin{aligned}
\sum_{1}=\sum_{1( \pm)} & =\sum_{l \leq m \leq d M} \sum_{p \leq x} I_{ \pm}\left(\frac{f(p)}{r^{m}}\right), \\
\sum_{2}=\sum_{2( \pm)} & =\sum_{d M<m \leq J-M} \sum_{1 \leq|\nu| \leq J^{2}} A_{ \pm}(\nu) \sum_{p \leq x} e\left(\frac{\nu}{r^{m}} f(p)\right), \\
\sum_{3}=\sum_{3( \pm)} & =\sum_{J-M<m \leq J} \sum_{1 \leq|\nu| \leq J^{2}} A_{ \pm}(\nu) \sum_{p \leq x} e\left(\frac{\nu}{r^{m}} f(p)\right) .
\end{aligned}
$$

We first estimate $\sum_{2}$. Suppose that $d M \leq m \leq J-M$. Then, writing the leading coefficient of the polynomial $\nu r^{-m} f(t)$ as $a / q$ with $(a, q)=1$, 
we have

$$
(\log x)^{\sigma} \leq q \leq x^{d}(\log x)^{-\sigma}
$$

with a large constant $\sigma$, so that by Lemma 2 ,

$$
\sum_{p \leq x} e\left(\frac{\nu}{r^{m}} f(p)\right) \ll x(\log x)^{-\sigma_{0}},
$$

where $\sigma_{0}>3$ is a constant. Therefore we obtain

$$
\sum_{2} \ll x(\log x)^{2-\sigma_{0}} \ll \frac{x}{\log x} .
$$

Next we estimate $\sum_{3}$. We appeal to the prime number theorem of the form referred to in Section 2. Then it follows that

$$
\begin{aligned}
\sum_{p \leq x} e\left(\frac{\nu}{r^{m}} f(p)\right) & =\int_{2}^{x} e\left(\frac{\nu}{r^{m}} f(t)\right) d \pi(t)+O(1) \\
& =\int_{2}^{x} e\left(\frac{\nu}{r^{m}} f(t)\right) \frac{d t}{\log t}+O\left(\frac{x}{(\log x)^{G}}\right) \\
& =\int_{x(\log x)^{-G}}^{x} e\left(\frac{\nu}{r^{m}} f(t)\right) \frac{d t}{\log t}+O\left(\frac{x}{(\log x)^{G}}\right) \\
& \ll \frac{1}{\log x} \sup _{\xi} \int_{x(\log x)^{-G}}^{\xi} e\left(\frac{\nu}{r^{m}} f(t)\right) d t \mid+O\left(\frac{x}{(\log x)^{G}}\right) \\
& \ll \frac{1}{\log x}\left(\frac{|\nu|}{r^{m}}\right)^{-1 / d}+O\left(\frac{x}{(\log x)^{G}}\right),
\end{aligned}
$$

using the second mean-value theorem and Lemma 1 with $\left|\nu r^{-m} f^{(d)}(t)\right| \gg$ $|\nu| r^{-m}$. Therefore we have

$$
\begin{aligned}
\sum_{3} & \ll \sum_{1 \leq|\nu| \leq J^{2}}|\nu|^{-1} \sum_{J-M \leq m \leq J}\left(\frac{1}{\log x}\left(\frac{|\nu|}{r^{m}}\right)^{-1 / d}+O\left(\frac{x}{(\log x)^{G}}\right)\right) \\
& \ll \frac{1}{\log x} \sum_{1 \leq|\nu| \leq J^{2}} \frac{1}{|\nu|^{1+1 / d}} \sum_{m \leq J} r^{-m / d}+O\left(\frac{x}{(\log x)^{G-2}}\right) \\
& \ll \frac{x}{\log x} .
\end{aligned}
$$

To prove the Theorem, it remains to show that

$$
\sum_{1}=\frac{\pi(x)}{r^{l}} d M+O\left(\frac{x}{\log x}\right),
$$


since this together with (4), (8), (9), and (10) implies

$$
\begin{aligned}
\sum_{p \leq x} N^{*}\left(f(p) ; b_{1} \ldots b_{l}\right) & =\frac{\pi(x)}{r^{l}} J+O(\pi(x)) \\
& =\frac{\pi(x)}{r^{l}} \log _{r} f(x)+O\left(\frac{x}{\log x}\right),
\end{aligned}
$$

which is the inequality (6).

5. Proof of Theorem (continued). We shall prove the inequality (11) in three steps.

First step. Suppose that $l \leq m \leq d M$, where $M$ is given by (7) with (4). We appeal to the prime number theorem for arithmetic progressions of the following form ([4; Sect. 17]): Let $\pi(x ; q, a)$ be the number of primes $p \leq x$ in an arithmetic progression $p \equiv a(\bmod q)$ with $(a, q)=1$ and let $\varphi(n)$ be the Euler function. Then

$$
\pi(x ; q, a)=\frac{1}{\varphi(q)} \operatorname{Li} x+O\left(x e^{-c \sqrt{\log x}}\right)
$$

uniformly in $1 \leq q \leq(\log x)^{H}$, where $c>0$ is a constant which depends on a constant $H>0$ given arbitrary. (A weaker result $O\left(x(\log x)^{-G}\right)$ is enough for our purpose.) Let $B$ denote the least common multiple of all denominators of the coefficients, other than the constant term, of $f(t)$. Then

$$
\begin{aligned}
\sum_{p \leq x} I_{ \pm}\left(\frac{f(p)}{r^{m}}\right)= & \sum_{\substack{p \leq x \\
(p, B r)=1}} I_{ \pm}\left(\frac{f(p)}{r^{m}}\right)+O(1) \\
= & \sum_{\substack{a \bmod B r^{m} \\
(a, B r)=1}} I_{ \pm}\left(\frac{f(a)}{r^{m}}\right) \pi\left(x ; B r^{m}, a\right)+O(1) \\
= & \sum_{\substack{a \bmod _{(a, B r)=1}\\
}} I_{ \pm}\left(\frac{f(a)}{r^{m}}\right)\left(\frac{1}{\varphi\left(B r^{m}\right)} \operatorname{Li} x+O\left(\frac{x}{(\log x)^{G}}\right)\right) \\
& +\frac{\pi(1)}{\varphi\left(B r^{m}\right)} \sum_{\substack{a \bmod B r^{m} \\
(a, B r)=1}} I_{ \pm}\left(\frac{f(a)}{r^{m}}\right)+O\left(r^{m} \frac{x}{(\log x)^{G}}\right) .
\end{aligned}
$$

Hence we have

$$
\sum_{1} \lesseqgtr \sum_{l \leq m \leq d M} \frac{\pi(x)}{\varphi\left(B r^{m}\right)} \sum_{\substack{a \bmod B r^{m} \\(a, B r)=1}} I_{ \pm}\left(\frac{f(a)}{r^{m}}\right)+O\left(M r^{d M} \frac{x}{(\log x)^{G}}\right)
$$




$$
\begin{aligned}
= & \sum_{l \leq m \leq d M} \frac{\pi(x)}{\varphi\left(B r^{m}\right)} \sum_{a \bmod B r^{m}} I_{ \pm}\left(\frac{f(a)}{r^{m}}\right) \sum_{b \mid(a, B r)} \mu(b)+O\left(\frac{x}{\log x}\right) \\
= & \sum_{b \mid B r} \mu(b) \sum_{l \leq m \leq d M} \frac{\pi(x)}{\varphi\left(B r^{m}\right)} \sum_{\substack{a \bmod B r^{m} \\
b \mid a}} I_{ \pm}\left(\frac{f(a)}{r^{m}}\right)+O\left(\frac{x}{\log x}\right) \\
= & \pi(x) \frac{B r}{\varphi(B r)} \sum_{b \mid B r} \mu(b) \sum_{l \leq m \leq d M} \frac{1}{B r^{m}} \sum_{1 \leq n \leq B r^{m} / b} I_{ \pm}\left(\frac{f(b n)}{r^{m}}\right) \\
& +O\left(\frac{x}{\log x}\right),
\end{aligned}
$$

where $\mu(n)$ is the Möbius function. Note that $B r=O(1)$.

Second step. We shall prove that, for each $b \mid B r$,

$$
\begin{aligned}
\sum_{l \leq m \leq d M} \frac{1}{B r^{m}} & \sum_{1 \leq n \leq B r^{m} / b} I_{ \pm}\left(\frac{f(b n)}{r^{m}}\right) \\
& =\sum_{l \leq m \leq d M} \frac{1}{B r^{M}} \sum_{1 \leq n \leq B r^{M} / b} I_{ \pm}\left(\frac{f(b n)}{r^{m}}\right)+O(1) .
\end{aligned}
$$

If $l \leq m \leq M$, then we have

$$
\frac{1}{B r^{m}} \sum_{1 \leq n \leq B r^{m} / b} I_{ \pm}\left(\frac{f(b n)}{r^{m}}\right)=\frac{1}{B r^{M}} \sum_{1 \leq n \leq B r^{M} / b} I_{ \pm}\left(\frac{f(b n)}{r^{m}}\right),
$$

so that

$$
\begin{aligned}
\sum_{l \leq m<M} \frac{1}{B r^{m}} \sum_{1 \leq n \leq B r^{m} / b} I_{ \pm}\left(\frac{f(b n)}{r^{m}}\right) & \\
& =\sum_{l \leq m \leq M} \frac{1}{B r^{M}} \sum_{1 \leq n<B r^{M} / b} I_{ \pm}\left(\frac{f(b n)}{r^{m}}\right) .
\end{aligned}
$$

If $d=1$, (14) implies (13). So in what follows we assume $d \geq 2$ and $M \leq$ $m \leq d M$. We have

$$
\begin{aligned}
& \sum_{1 \leq n \leq B r^{m} / b} I_{ \pm}\left(\frac{f(b n)}{r^{m}}\right) \\
\lessgtr & \frac{B r^{m}}{b} \cdot \frac{1}{r^{l}}+O\left(\frac{r^{m}}{J}\right)+O\left(\left.\sum_{1 \leq|\nu| \leq J^{2}} \frac{1}{|\nu|}\right|_{1 \leq n \leq B r^{m} / b} e\left(\frac{\nu}{r^{m}} f(b n)\right) \mid\right) \\
= & \frac{B r^{m}}{b} \cdot \frac{1}{r^{l}}+O\left(\frac{r^{m}}{J}\right)+O\left(r^{m(1-1 / d)} J^{2 / d} \log J\right),
\end{aligned}
$$


since, by Lemma 3,

$$
\left|\sum_{1 \leq n \leq B r^{m} / b} e\left(\frac{\nu}{r^{m}} f(b n)\right)\right| \ll\left(r^{m}, \nu\right)^{1 / d} r^{m(1-1 / d)} .
$$

Hence we get

$$
\sum_{M \leq m \leq d M} \frac{1}{B r^{m}} \sum_{1 \leq n \leq B r^{m} / b} I_{ \pm}\left(\frac{f(b n)}{r^{m}}\right)=\frac{(d-1) M}{b r^{l}}+O(1) .
$$

In the rest of this step, we shall prove the inequality

$$
\sum_{M \leq m \leq d M} \frac{1}{B r^{M}} \sum_{1 \leq n \leq B r^{M} / b} I_{ \pm}\left(\frac{f(b n)}{r^{m}}\right)=\frac{(d-1) M}{b r^{l}}+O(1)
$$

which together with (15) and (14) yields (13).

Proof of (16). It is easily seen that

$$
\begin{aligned}
& \sum_{M \leq m \leq d M} \frac{1}{B r^{M}} \sum_{1 \leq n \leq B r^{M} / b} I_{ \pm}\left(\frac{f(b n)}{r^{m}}\right) \\
& \leq \frac{1}{B r^{M}} \sum_{M \leq m \leq d M} \sum_{1 \leq n \leq B r^{M} / b}\left(\frac{1}{r^{l}}+O\left(\frac{1}{J}\right)\right. \\
& \left.+\sum_{1 \leq|\nu| \leq J^{2}} A_{ \pm}(\nu) e\left(\frac{\nu}{r^{m}} f(b n)\right)\right) \\
& =\frac{(d-1) M}{b r^{l}}+O(1) \quad \\
& \quad+O\left(\sum_{1 \leq|\nu| \leq J^{2}} \frac{1}{|\nu|} \cdot \frac{1}{B r^{M}} \sum_{M \leq m \leq d M}\left|\sum_{1 \leq n \leq B r^{M} / b} e\left(\frac{\nu}{r^{m}} f(b n)\right)\right|\right) .
\end{aligned}
$$

We estimate the last sum. Let $H$ be a large constant. For any $\nu, m, b$, we can choose, by Dirichlet's theorem, coprime integers $a$ and $q=q(\nu, m, b)$ such that

$$
1 \leq q \leq Q^{d} /(\log Q)^{H}, \quad Q=B r^{M} / b
$$

and

$$
\left|\frac{\nu}{r^{m}} b^{d}-\frac{a}{q}\right|<\frac{(\log Q)^{H}}{q Q^{d}} \quad\left(\leq 1 / q^{2}\right)
$$

If

$$
(\log Q)^{H} \leq q \leq Q^{d} /(\log Q)^{H}
$$


then by Lemma 4 ,

$$
\left|\sum_{1 \leq n \leq B r^{M} / b} e\left(\frac{\nu}{r^{m}} f(b n)\right)\right| \ll \frac{Q}{(\log Q)^{G}} \ll \frac{r^{M}}{(\log J)^{2}} .
$$

Hence the contribution of these sums in the last term in (17) is

$$
\ll \frac{1}{B r^{M}}(d-1) M \log J \cdot \frac{r^{M}}{(\log J)^{2}}=O(1) .
$$

Otherwise, we have

$$
1 \leq q \leq(\log Q)^{H} \quad\left(\gg \ll M^{H}\right) .
$$

In particular, $\left(\nu / r^{m}\right) b^{d} \neq a / q$, since $m \geq M$. Hence

$$
\frac{1}{q r^{m}} \leq\left|\frac{\nu}{r^{m}} b^{d}-\frac{a}{q}\right| \ll \frac{M^{H}}{q r^{d M}},
$$

so that

$$
(d M \geq) m \geq d M-H_{1} \log M,
$$

with a large constant $H_{1}$. From this it follows that

$$
\frac{d}{d t} \cdot \frac{\nu}{r^{m}} f(b t) \gg \ll \frac{\nu}{r^{m}} t^{d-1} \ll J^{2} r^{-M+H_{1} \log M}=o(1)
$$

throughout the interval $\left[1, B r^{M} / b\right]$. Thus by a van der Corput's lemma ([9; Lemma 4.8]) we have

$$
\begin{aligned}
\sum_{1 \leq n \leq B r^{M} / b} e\left(\frac{\nu}{r^{m}} f(b n)\right) & =\int_{1}^{B r^{M} / b} e\left(\frac{\nu}{r^{m}} f(b t)\right) d t+O(1) \\
& \ll\left|\frac{\nu}{r^{m}} f^{(d)}(t)\right|^{-1 / d}+O(1) \ll\left(\frac{|\nu|}{r^{m}}\right)^{-1 / d},
\end{aligned}
$$

using again Lemma 1. Hence the contribution of these sums to the last term in $(17)$ is

$$
\ll \frac{1}{B r^{M}} \sum_{M \leq m \leq d M} \sum_{1 \leq|\nu| \leq J^{2}} \frac{1}{|\nu|}\left(\frac{|\nu|}{r^{m}}\right)^{-1 / d}=O(1) .
$$

Combining these results, we obtain (16). 
Third step. It follows from (12) with (13) that

$$
\begin{aligned}
\sum_{1} \lesseqgtr & \pi(x) \frac{B r}{\varphi(B r)} \sum_{b \mid B r} \mu(b) \frac{1}{B r^{M}} \sum_{l \leq m \leq d M} \sum_{1 \leq n \leq B r^{M} / b} I_{ \pm}\left(\frac{f(b n)}{r^{m}}\right) \\
& +O\left(\frac{x}{\log x}\right) \\
\lesseqgtr & \pi(x) \frac{B r}{\varphi(B r)} \sum_{b \mid B r} \mu(b) \frac{1}{B r^{M}} \sum_{l \leq m \leq d M} \sum_{1 \leq n \leq B r^{M} / b} I\left(\frac{f(b n)}{r^{m}}\right) \\
& +O\left(\frac{x}{\log x}\right) .
\end{aligned}
$$

We put, in Lemma $5, y=B r^{M} / b$, so that $\log _{r} f(b y)=d M+O(1)$. Then we have

$$
\begin{aligned}
\sum_{l \leq m \leq d M} \sum_{1 \leq n \leq B r^{M} / b} I\left(\frac{f(b n)}{r^{m}}\right) & =\sum_{n \leq y} N\left(f(b n) ; b_{1} \ldots b_{l}\right)+O\left(r^{M}\right) \\
& =r^{-l} y \log _{r} f(b y)+O\left(r^{M}\right) \\
& =r^{-l} \frac{B r^{M}}{b} d M+O\left(r^{M}\right) .
\end{aligned}
$$

Therefore we obtain

$$
\begin{aligned}
\sum_{1} & \gtreqless \frac{B r}{\varphi(B r)} \sum_{b \mid B r} \frac{\mu(b)}{b} \cdot \frac{d M}{r^{l}} \pi(x)+O\left(\frac{x}{\log x}\right) \\
& =r^{-l} d M \pi(x)+O\left(\frac{x}{\log x}\right),
\end{aligned}
$$

which is (11). The proof of the Theorem is now complete.

\section{References}

[1] A. H. Copeland and P. Erdős, Notes on normal numbers, Bull. Amer. Math. Soc. 52 (1946), 857-860.

[2] H. Daven port and P. Erdős, Note on normal decimals, Canad. J. Math. 4 (1952), $58-63$.

[3] L.-K. Hua, Additive Theory of Prime Numbers, Transl. Math. Monograph 13, Amer. Math. Soc., Providence, RI, 1965.

[4] M. N. Huxley, The Distribution of Prime Numbers, Oxford Math. Monograph, Oxford Univ. Press, 1972.

[5] Y.-N. Nakai and I. Shiokawa, A class of normal numbers, Japan. J. Math. 16 (1990), 17-29.

[6] - - - A class of normal numbers II, in: Number Theory and Cryptography, J. H. Loxton (ed.), London Math. Soc. Lecture Note Ser. 154, Cambridge Univ. Press, 1990, 204-210. 
[7] Y.-N. Nakai and I. Shiokawa, Discrepancy estimates for a class of normal numbers, Acta Arith. 62 (1992), 271-284.

[8] J. Schiffer, Discrepancy of normal numbers, ibid. 47 (1986), 175-186.

[9] E. C. Titchmarsh, The Theory of the Riemann Zeta-Function, 2nd ed., revised by D. R. Heath-Brown, Oxford Univ. Press, 1986.

[10] I. M. Vinogradov, The Method of Trigonometrical Sums in Number Theory, Nauka, 1971 (in Russian).

Department of Mathematics

Faculty of Education

Yamanashi University

Kofu, 400 Japan

E-mail: nakai@grape.kkb.yamanashi.ac.jp
Department of Mathematics Keio University Hiyoshi, Yokohama, 223 Japan E-mail: shiokawa@math.keio.ac.jp

Received on 28.6.1996

and in revised form on 16.12.1996 